1

3

7 james.bartlett@glasgow.ac.uk

\section{No Meaningful Difference in Attentional Bias Between Daily and Non-Daily Smokers} James E Bartlett ${ }^{1}$, Rebecca Jenks ${ }^{1}$, \& Nigel Wilson ${ }^{1}$

${ }^{1}$ School of Psychological, Social, and Behavioural Sciences, Coventry University

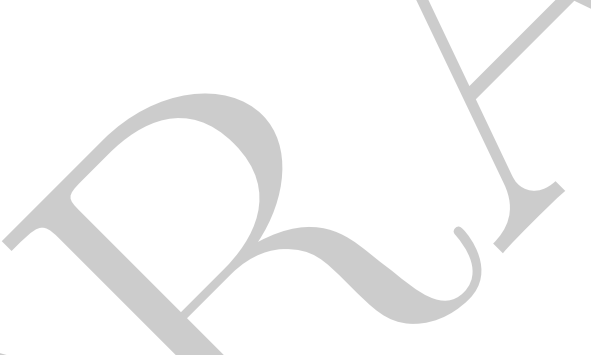

Author Note

Correspondence concerning this article should be addressed to James E Bartlett, 62 Hillhead Street, University of Glasgow, Glasgow, United Kingdom. E-mail: 
9

Abstract

Both daily and non-daily smokers find it difficult to quit smoking long-term. One factor associated with addictive behaviour is attentional bias, but previous research in daily and non-daily smokers found inconsistent results and did not report the reliability of their cognitive tasks. Using an online sample, we compared daily $(\mathrm{n}=106)$ and non-daily $(\mathrm{n}=$ 60) smokers in their attentional bias towards smoking pictures. Participants completed a visual probe task with two picture presentation times: 200ms and 500ms. In confirmatory analyses, there were no significant effects of interest, and in exploratory analyses, equivalence testing showed the effects were statistically equivalent to zero. The reliability of the visual probe task was poor, meaning it should not be used for repeated testing or investigating individual differences. The results can be interpreted in line with contemporary theories of attentional bias where there are unlikely to be stable trait-like differences between smoking groups. Future research in attentional bias should focus on state-level differences using more reliable measures than the visual probe task.

Keywords: Non-daily smokers, Daily smokers, Visual probe task, Attentional bias,

Equivalence testing Word count: 4854

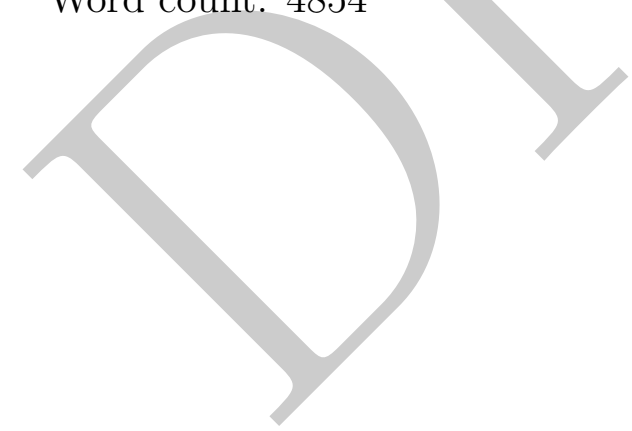


No Meaningful Difference in Attentional Bias Between Daily and Non-Daily Smokers

\section{Take-home message}

Previous research reported greater attentional bias in daily or non-daily smokers using the visual probe task. We found no meaningful difference using the traditional approach of analysing differences in response times. Our visual probe task also showed poor reliability, meaning response time outcomes from the task should not be used in individual differences research or measuring changes in attentional bias across repeated measurements.

\section{Purpose}

Daily and non-daily smokers have different habits and motives but both groups find it difficult to quit smoking long-term. As attentional bias may be associated with addictive behaviour, we used the visual probe task to compare daily and non-daily smokers. We predicted that non-daily smokers would show greater attentional bias towards smoking images than daily smokers. If non-daily smokers showed greater attentional bias, it would help to explain why they find it difficult to quit smoking while showing fewer signs of nicotine dependence.

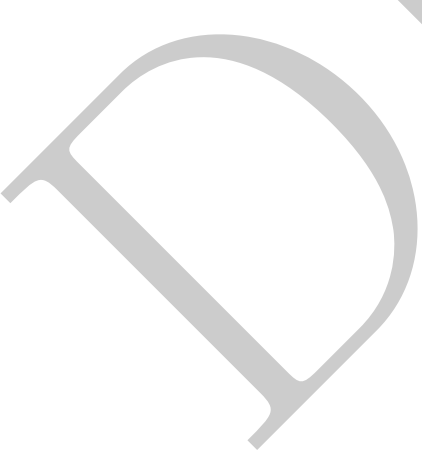




\section{Introduction}

Historically, smokers have been treated as a single homogeneous group (Shiffman, 2009), but there are fundamental differences in the smoking habits and motives of daily and non-daily smokers (Shiffman, Dunbar, et al., 2012; Shiffman, Tindle, et al., 2012). 13-36\% of smokers are defined as non-daily smokers across Europe and the United States (Bogdanovica et al., 2011; Kotz et al., 2012; Tindle \& Shiffman, 2011) and non-daily smoking has typically been the most prevalent pattern in ethnic minority groups (Fagan \& Rigotti, 2009; Tong et al., 2006). Whereas daily smokers cite negative reinforcers such as avoiding nicotine withdrawal as the key motivators, non-daily smokers cite positive reinforcers such as smoking around friends and alcohol (Shiffman et al., 2014; Shiffman, Dunbar, et al., 2012). Despite these differences, $77-92 \%$ of daily smokers and $74-83 \%$ of non-daily smokers relapse within 90 days of a quit attempt (Bogdanovica et al., 2011; Kotz et al., 2012; Tindle \& Shiffman, 2011), showing both groups find it difficult to quit smoking long-term. This means it is important to investigate potential factors associated with smoking behaviour.

One factor is attentional bias, which is the tendency to fixate attention on cues associated with smoking. Attentional bias is the product of a classical conditioning process where smokers develop conditioned responses to substance-related cues through repeated exposure (Field \& Cox, 2008). Theoretical models of attentional bias suggest it has a reciprocal relationship with craving, supported by a meta-analysis showing there is a small positive relationship (Field et al., 2009). In situations where cigarettes are available, cues associated with smoking grab attention, induce craving, which further drives attentional bias. Updated theories of attentional bias (Field et al., 2016) emphasise the role of momentary evaluations of smoking cues, meaning attentional bias and craving fluctuates over time, and describe attempts to extinguish the conditioned response through attentional bias modification. Due to its predicted role in smoking behaviour, studies have investigated whether attentional bias is higher in certain groups or conditions. 
Consistent with theoretical models, smokers consistently show greater attentional bias towards smoking cues than non-smokers (Baschnagel, 2013; Ehrman et al., 2002; Kang et al., 2012; Mogg et al., 2003). However, there are contrasting expectations and findings around how lighter and heavier smokers ${ }^{1}$ differ in attentional bias. One view is lighter smokers should show greater attentional bias than heavier smokers since they rarely show signs of nicotine dependence, so smoking-related cues are required to induce craving and motivate substance use. In support, some studies found lighter smokers exhibit greater attentional bias than heavier smokers (Bradley et al., 2003; Hogarth et al., 2003; Mogg et al., 2005). Another view is heavier smokers should show greater attentional bias than lighter smokers since the conditioned response to smoking-related cues should be stronger from repeated exposure. There is also evidence for this view as heavier smokers showed greater attentional bias than lighter smokers (Chanon et al., 2010; Vollstädt-Klein et al., 2011; Zack et al., 2001). Collectively, these studies show smokers consistently display greater attentional bias towards smoking cues than non-smokers, but it is not clear whether lighter or heavier smokers show greater attentional bias.

To address this inconsistency, the current study focused on comparing attentional bias towards smoking cues in daily and non-daily smokers. Despite most studies using the visual probe task to measure attentional bias, relatively small sample sizes and inconsistent design features complicate making conclusions about the mixed findings. Therefore, we used a much larger sample size than previous studies and manipulated different features of the visual probe task.

The visual probe task infers attention through differences in response time (RT). Two images are presented and when they disappear, the participant is required to indicate the location of a small probe that replaces one of the images. Faster RTs to particular stimuli

\footnotetext{
${ }^{1}$ Note, we refer to lighter and heavier smokers here as the studies used different definitions. In our study, we operationalise the groups as daily and non-daily smokers.
} 
reflect selective attention (Field \& Cox, 2008), but as the location of attention is inferred through differences in RT after the stimuli disappear, the presentation time can be manipulated. Short Stimulus Onset Asynchronies (SOA) of 200ms or less measure involuntary attentional processes (Field \& Cox, 2008). Longer SOAs of 500ms or more target voluntary attention as there is enough time to make multiple fixations. Previous research used single SOAs of 500ms (Vollstädt-Klein et al., 2011) and 2000ms (Hogarth et al., 2003; Mogg et al., 2005). None of the studies used a very short SOA to measure more involuntary attentional processes. Chanon et al. (2010) found that in comparison to non-smokers, attentional bias was greater in smokers under a $200 \mathrm{~ms}$ conditions than a $550 \mathrm{~ms}$ condition. To investigate the conflict in results between daily and non-daily smokers, this study used two SOAs of $200 \mathrm{~ms}$ and $500 \mathrm{~ms}$.

A final consideration of our study was to evaluate and report the internal consistency of the visual probe task. There is growing awareness that the reliability of cognitive tasks should be taken seriously (Parsons et al., 2019; Pennington et al., 2021), but reliability has a different meaning depending on the context. For experimental measures to be reliable, we want to consistently observe effects between groups or conditions, but for correlational measures to be reliable, we want to consistently rank individuals (Hedge et al., 2018). This means the attributes of experimental measures may not be compatible with the requirements for reliable correlation research. As researchers often use the visual probe task as a measure in cognitive bias modification procedures, it must be reliable to detect any changes across time. Previous attempts at evaluating the internal consistency of the visual probe task have been disappointing (Ataya et al., 2012; Schmukle, 2005; Waechter et al., 2014). Therefore, we are following recommendations to habitually report the reliability of cognitive tasks (Parsons et al., 2019), even when it is not the main focus of the study.

The protocol and hypotheses for this project were pre-registered on the Open Science Framework (OSF; https://osf.io/ju7kv). Given the relevance of smoking cues for non-daily 
smokers and the results from previously unpublished research, we hypothesised non-daily smokers would show greater attentional bias than daily smokers. There was no a priori hypothesis for the effect of SOA condition. This means we expected non-daily smokers to show greater attentional bias than daily smokers, but it was not clear what the difference in magnitude would be under different SOA conditions.

\section{Method}

\section{Design}

We used a 2 × 2 mixed design with one between-subjects IV of smoking group with two levels: daily and non-daily smokers. Participants responded to the question "Do you usually smoke cigarettes every day?". Non-daily smokers responded "No" and daily smokers responded "Yes". There was one within-subjects IV of the visual probe task SOA which had two levels: $200 \mathrm{~ms}$ and $500 \mathrm{~ms}$. The dependent variable was the attentional bias index (ms) calculated by subtracting the mean RT to smoking trials from the mean RT to non-smoking trials. This means positive values indicate greater attentional bias towards smoking cues.

\section{Participants and Sample Size Calculation}

We collected data online using Prolific where inclusion criteria consisted of participants should have normal or corrected-normal vision, be between the ages of 18 to 60 , and smoke at least one cigarette per week or four cigarettes per month.

We simulated a power analysis to justify the sample size. We set the smallest effect size of interest based on a previously unpublished study (Bartlett, 2020) where the mean difference in attentional bias score between smoking groups was $6.13 \mathrm{~ms}$ (95\% $\mathrm{CI}=[-5.27$, $17.53])$ for a $200 \mathrm{~ms} \mathrm{SOA}$ and $11.35 \mathrm{~ms}(95 \% \mathrm{CI}=[-4.51,27.21]$ for a $500 \mathrm{~ms} \mathrm{SOA}$. However, we also consulted previous research due to the wide confidence intervals. The smallest known effects for a $200 \mathrm{~ms}$ SOA was $5 \mathrm{~ms}$ (Chanon et al., 2010) and 11ms for a 500ms SOA (Bradley et al., 2003). Our smallest effect sizes of interest were $5 \mathrm{~ms}(200 \mathrm{~ms})$ and $10 \mathrm{~ms}(500 \mathrm{~ms})$, and a 
conservative standard deviation of 20ms based on Vollstädt-Klein et al. (2011).

These values were used to conduct a simulated power analysis for a $2 \times 2$ mixed ANOVA using R (code available on the OSF; https://osf.io/am9hd/). We expected non-daily smokers to display greater attentional bias towards smoking images than daily smokers. We set the conditions of the power analysis as non-daily smokers having a $5 \mathrm{~ms}$ (200ms) and $10 \mathrm{~ms}(500 \mathrm{~ms})$ greater mean difference than daily smokers. For each condition, the values were sampled from a normal distribution with a standard deviation of $20 \mathrm{~ms}$. The sample size for each smoking group was increased from $10(\mathrm{~N}=20)$ to $150(\mathrm{~N}=300)$ in steps of 10 , with each step repeating 10,000 times. The final sample size target was 60 per group $(\mathrm{N}=120)$ as we reached $80 \%$ power (alpha $=.05)$ between 50 and 60 participants per group.

\section{Materials}

Fagerström Test for Cigarette Dependence (FTCD). The FTCD (Fagerström, 2012; Heatherton et al., 1991) was used as a self-report measure of nicotine dependence. The Cronbach's alpha estimate (bootstrapped using 10,000 iterations) in this sample was higher than in previous research, $\alpha=74,95 \% \mathrm{CI}=[.67, .8]$.

Visual Probe Task. We used Gorilla (Anwyl-Irvine et al., 2019) to present the visual probe task online and the task is available on the open materials page to preview or clone (http://gorilla.sc/openmaterials/85021).

Each trial started with a $250 \mathrm{~ms}$ central fixation cross before two images were presented horizontally to the left and right. The content and duration of the two images was controlled by two variables: trial type and SOA. Trial type consisted of three conditions (neutral, smoking, or non-smoking) while SOA consisted of two conditions (200ms or $500 \mathrm{~ms})$. At picture offset, a small dot appeared in the location vacated by one of the images. The dot remained on the screen until the participant responded either left (Z key) or right (M key). After they responded, the next trial began with the screen containing only the fixation cross. 
The trial procedure is shown visually in Figure 1.

The trial type condition was based on 16 image pairs for neutral trials and 16 image pairs for smoking and non-smoking trials. For neutral trials, the dot replaced one of the neutral image pairs. For smoking trials, the dot replaced a smoking image presented next to a matched non-smoking image. For non-smoking trials, the dot replaced a non-smoking image presented next to a smoking image.

We used 16 image pairs from the International Affective Picture System (Lang et al., 2008) for the neutral trials. We developed a series of matching smoking and non-smoking images for the smoking and non-smoking trials (Bartlett, 2020). The list of IAPS images is available on the OSF project and our smoking/non-smoking images are available on the Gorilla open materials page.

The trial order was randomised with each picture pair presented four times to cover each combination of image (left and right) and dot location (left and right). This combination determined the trial type condition, where a left smoking image, right non-smoking image, and left dot would produce a smoking trial. For each picture pair, this process was repeated twice for each SOA condition, producing 384 trials split into two blocks with 64 trials in each SOA and trial type condition.

\section{Procedure}

We provided participants with an information sheet and they provided informed consent by ticking a box. This study was approved by the Faculty of Health and Life Sciences Ethical Approval board. Participants completed a short questionnaire on their demographic information, smoking habits, and the FTCD. The next page contained the visual probe task which began with a set of instructions asking the participant to complete the task in a quiet environment free of distractions. Participants completed 12 practice trials which provided feedback on their responses and overall accuracy. After the task, participants 


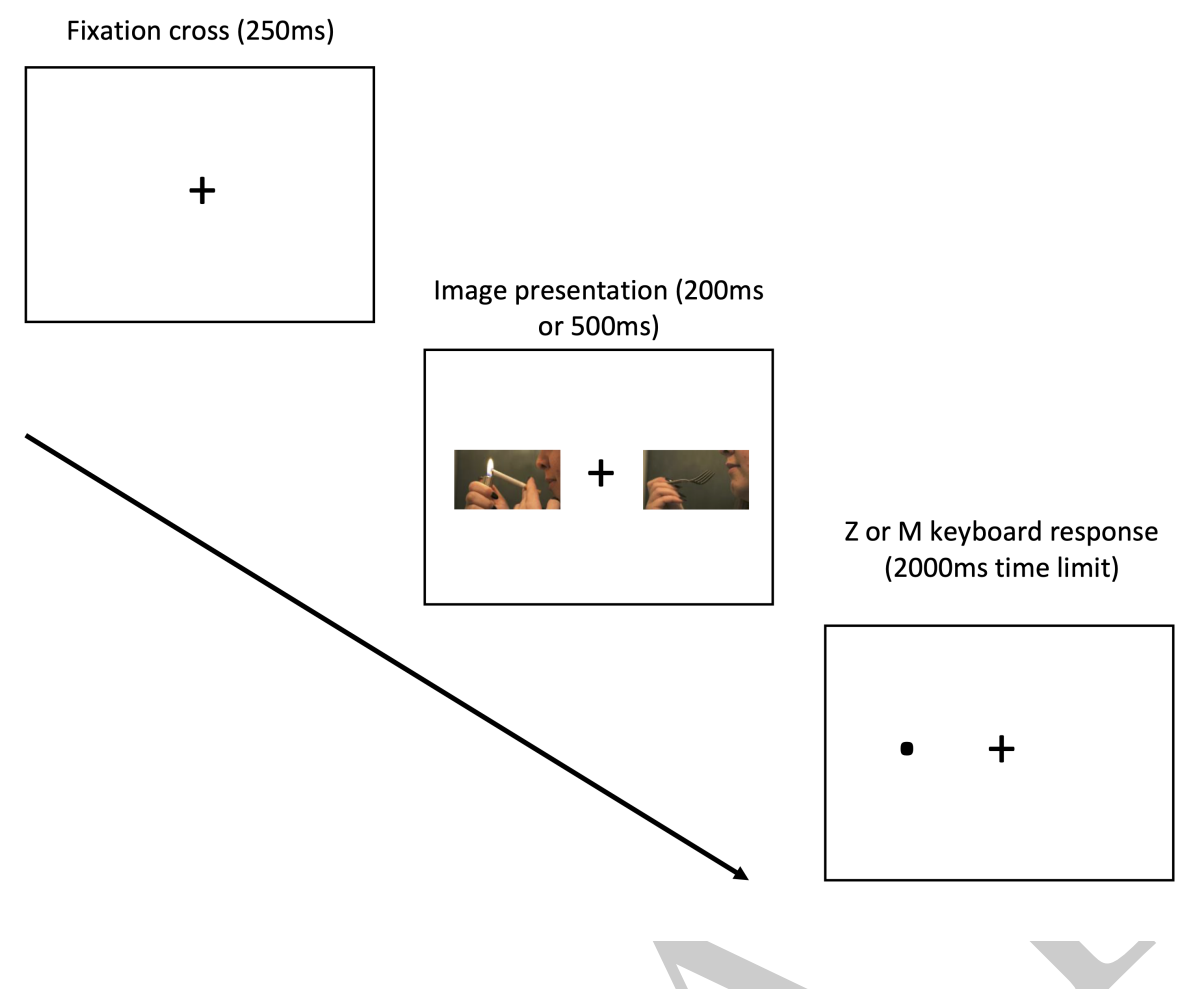

Figure 1. Diagram showing the trial procedure of the visual probe task. Each trial started with a fixation cross lasting $250 \mathrm{~ms}$. The fixation cross is then flanked by one of the stimulus pairs on the left and right. The stimuli remained on the screen for $200 \mathrm{~ms}$ or $500 \mathrm{~ms}$ depending on the SOA condition. The stimuli disappear and one image is replaced with a small dot. Participants had up to $2000 \mathrm{~ms}$ to respond whether the dot was on the left or right. The next trial started with a new blank fixation cross. device, and if they had completed the study before. Similar to Clifford and Jerit (2014), we asked participants if they had any distractions while they completed the study such as listening to music. Finally, participants read a debriefing sheet before they were redirected to Prolific. If the participants successfully reached the end of the study, they were paid $£ 2$.

\section{Results}


Table 1

Mean (SD) values for participant characteristics and scale scores.

\begin{tabular}{lll}
\hline & Non-Daily Smokers & Daily Smokers \\
\hline Age & $28.68(7.71)$ & $31.84(9.7)$ \\
$\%$ female & $46.67 \%$ & $26.42 \%$ \\
$\%$ white & $93 \%$ & $92 \%$ \\
FTCD & $0.52(1.31)$ & $2.58(2.17)$ \\
Cigarettes per day & $2.38(2.74)$ & $8.59(6.41)$ \\
Age started to smoke & $18.51(3.65)$ & $17.93(3.47)$ \\
Time since last cigarette (minutes) & $2880(4590)$ & $60(633.75)$ \\
\hline
\end{tabular}

Note. *Due to large skew, these values represent the median and IQR.

\section{Participant Attrition and Demographics}

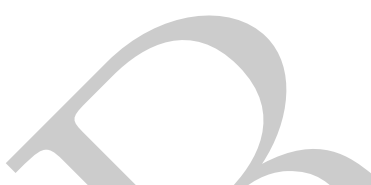

218 people accessed the study and 205 completed the experiment and received payment. The final sample was 166 after applying exclusion criteria: 60 non-daily and 106 daily smokers. Participants were excluded for having fewer than $50 \%$ of the possible trials (n $=4)$, experiencing technical issues $(\mathrm{n}=16)$, reporting to smoke every day but not every week $(\mathrm{n}=3)$, and not smoking in the past four weeks $(\mathrm{n}=19)$. The total number equals 42 as some participants met more than one criterion.

Table 1 displays the demographic information. Daily smokers smoked more cigarettes per day and had a higher FTCD score. Non-daily smokers exemplified infrequent smoking as the median time since their last cigarette was 48 hours, while it was only one hour for daily smokers. Figure 2 shows the distribution of FTCD scores and cigarettes per day. 

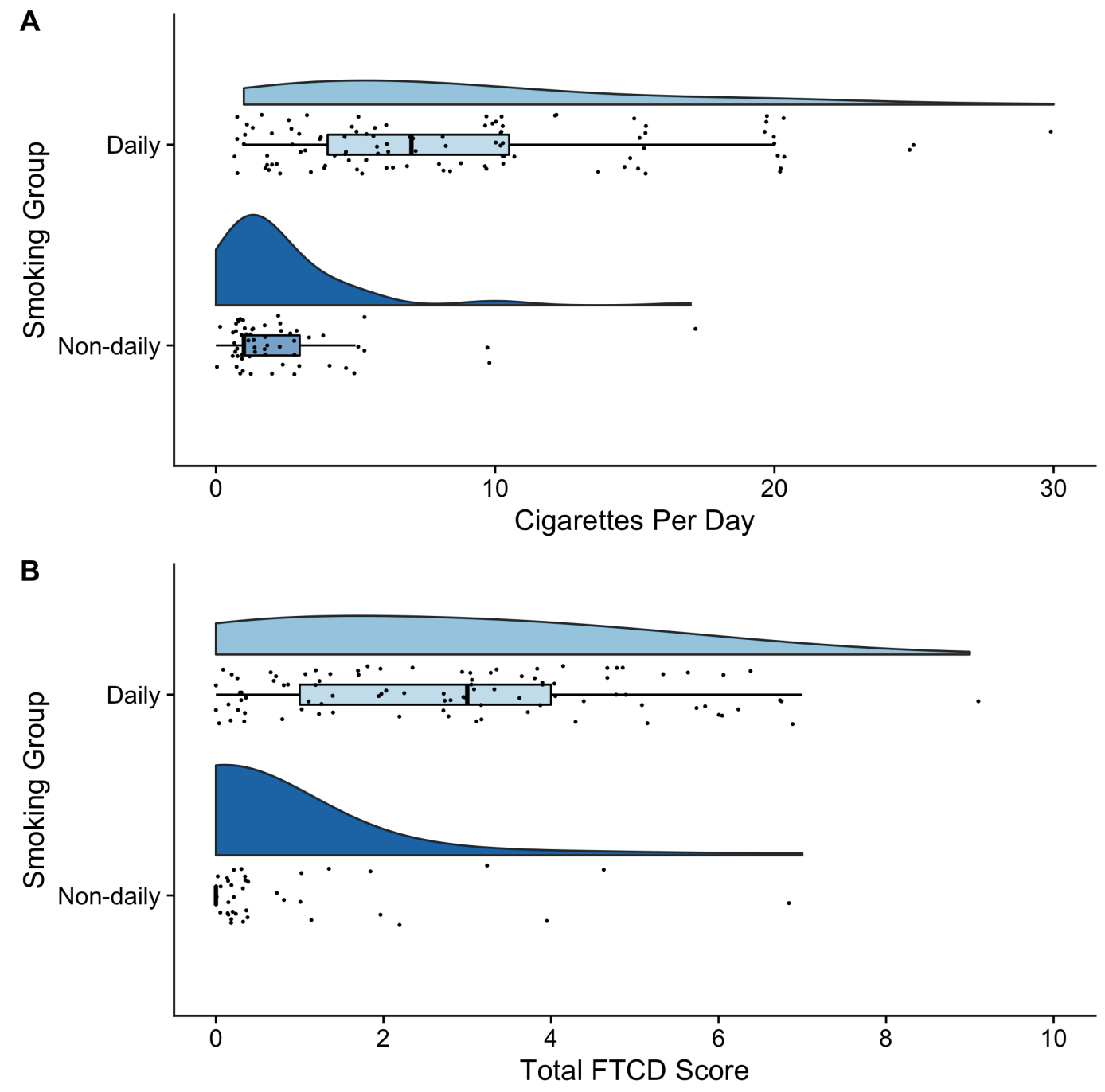

Figure 2. Two different measures of nicotine dependence: (A) number of cigarettes per day and (B) FTCD score. The data are presented as raincloud plots (Allen et al., 2019). The top element for each group represents the distribution of scores through the density. The bottom element presents the individual data points with a superimposed boxplot. 


\section{Data Processing}

The $\mathrm{R}$ code is available on the OSF (https://osf.io/am9hd/). We removed incorrect responses in addition to responses faster than 200ms as they represent preemptive responses. We considered outliers as any response outside 2.5 times the median absolute deviation for each participant, SOA, and trial condition (Leys et al. 2013). This meant we removed 9.72\% of the total possible trials, with the median number of excluded trials for each participant being 23 (range $7-98$ ).

For the confirmatory analyses, we focused on smoking/non-smoking image pairs and excluded the neutral pairs. Originally, we planned on conducting exploratory analyses to create orienting and disengagement indices (Salemink et al., 2007) by subtracting the mean RT to neutral trials from smoking trials (orienting) or non-smoking trials (disengagement), but a coding error meant we did not have matching numbers of neutral trials in the $200 \mathrm{~ms}$ and 500ms SOA conditions. Therefore, we focused on our confirmatory analyses and excluded neutral trials.

After removing outliers, we calculated the mean RT to probes that replaced non-smoking images and the mean RT to probes that replaced smoking images. We then calculated the difference between these two values as our attentional bias index (non-smoking - smoking), where positive values mean faster average responses to smoking images. For each participant, this produced two values: one for the attentional bias index using a 200ms SOA and one using a 500ms SOA.

\section{Confirmatory Analyses: Attentional Bias Towards Smoking Cues}

The mean $(S D)$ attentional bias index in the 200ms SOA condition was $1.95 \mathrm{~ms}(22.31)$ for daily smokers and -0.30ms (18.57) for non-daily smokers. In the 500ms SOA condition, the mean bias index was $0.21 \mathrm{~ms}$ (21.93) for daily smokers and -2.06ms (12.67) for non-daily smokers. This was in the opposite direction to our hypotheses as we expected non-daily 

results are displayed in Figure 3.

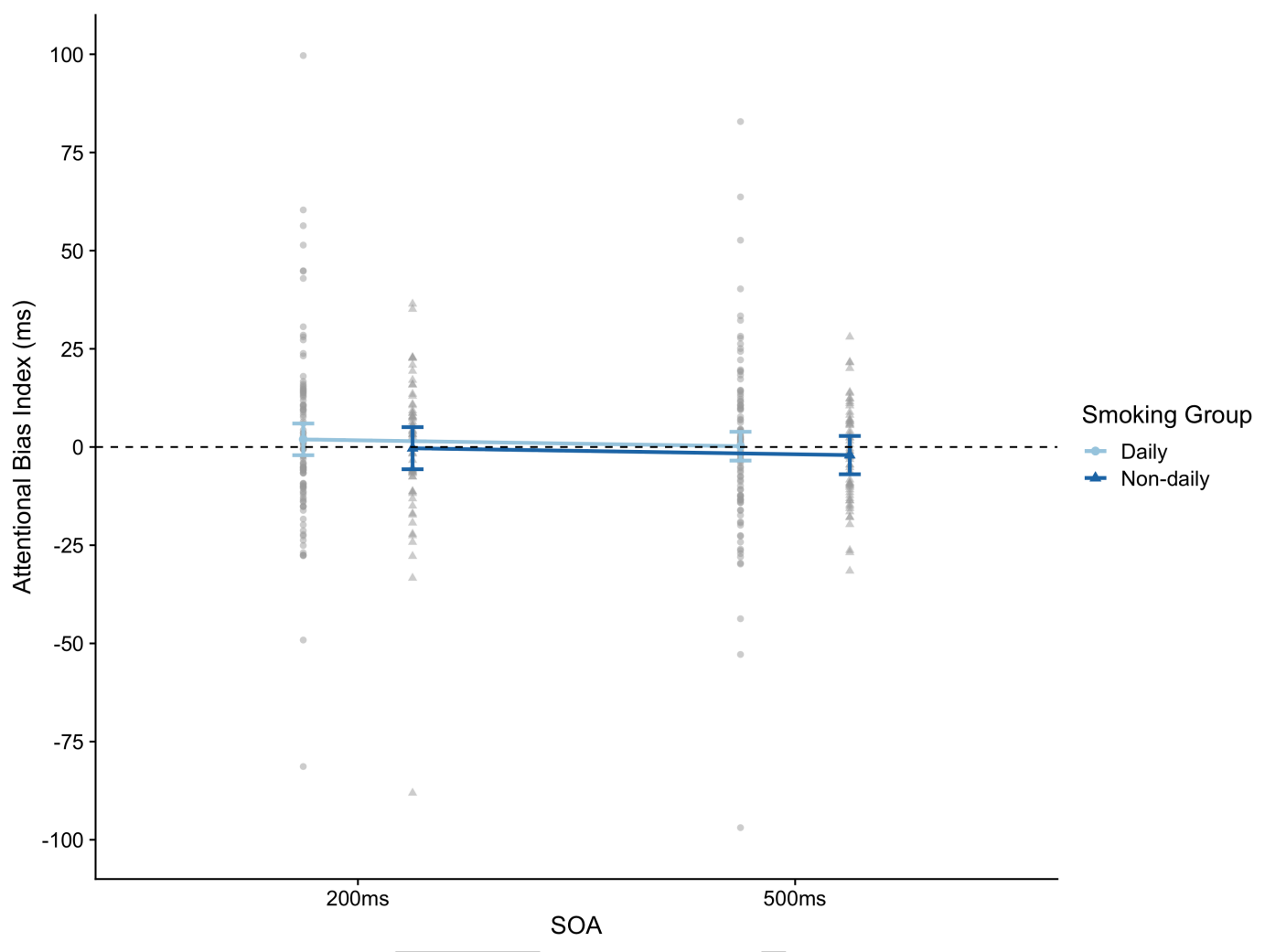

smokers to display greater attentional bias towards smoking images than daily smokers. The

Figure 3. Interaction plot showing the mean attentional bias index for daily and non-daily smokers by SOA condition. The error bars represent the 95\% CI around the mean. Positive values indicate greater attentional bias towards smoking cues. The grey points show the individual scores per condition.

We used a 2x2 mixed ANOVA with SOA as a within-subjects IV and smoking group as a between-subjects IV. The mean attentional bias index was the DV. There was not a 


\section{Exploratory Analyses: No Meaningful Difference in Attentional Bias}

To demonstrate there was no meaningful difference between daily and non-daily smokers, we performed equivalence testing on the two comparisons of interest: the difference between daily and non-daily smokers at each SOA condition. You cannot directly provide evidence in favour of the null hypothesis using traditional null hypothesis significance testing. Equivalence testing applies two one-sided tests to user-defined boundaries representing effects you consider too small to be practically or theoretically meaningful (Lakens et al., 2018). If both tests are statistically significant, you can conclude your observed effect size is statistically equivalent to zero based on your boundaries.

There are different approaches to setting the boundaries for your smallest effect size of interest. We used Cohen's $d= \pm 0.41$ based on the small telescopes method (Lakens et al., 2018). This is where you use the effect size that the largest previous study had $33 \%$ power to detect (in our case, Vollstädt-Klein et al. (2011) with two groups of 25 and 26 participants). The small telescopes method is appropriate when previous research did not define their smallest effect size of interest, so it represents the effect size large enough to be detectable in the original study (Simonsohn, 2015). Considering alternative choices for the effect size boundaries, our conclusions below hold when we use the larger effect size from our power analysis (10ms) but not when we use the smaller effect size (5ms). Given we are arguing differences in attentional bias in daily and non-daily smokers may be smaller than reported in previous research, we focus on the results using the small telescopes method.

For the $200 \mathrm{~ms}$ SOA condition, the two one-sided test procedure was significant, demonstrating that the difference in attentional bias towards smoking images between daily and non-daily smokers was statistically equivalent to zero, $t(141.65)=-1.91, p=.029$. Similarly, the 500ms SOA condition was statistically equivalent to zero, $t(163.91)=-1.89, p$ $=.03$. The equivalence testing procedure is presented in Figure 4, showing that the $90 \%$ confidence interval around the mean difference crosses zero, but does not cross the effect size 
boundaries of $\mathrm{d}= \pm .41$ (expressed here in raw units).

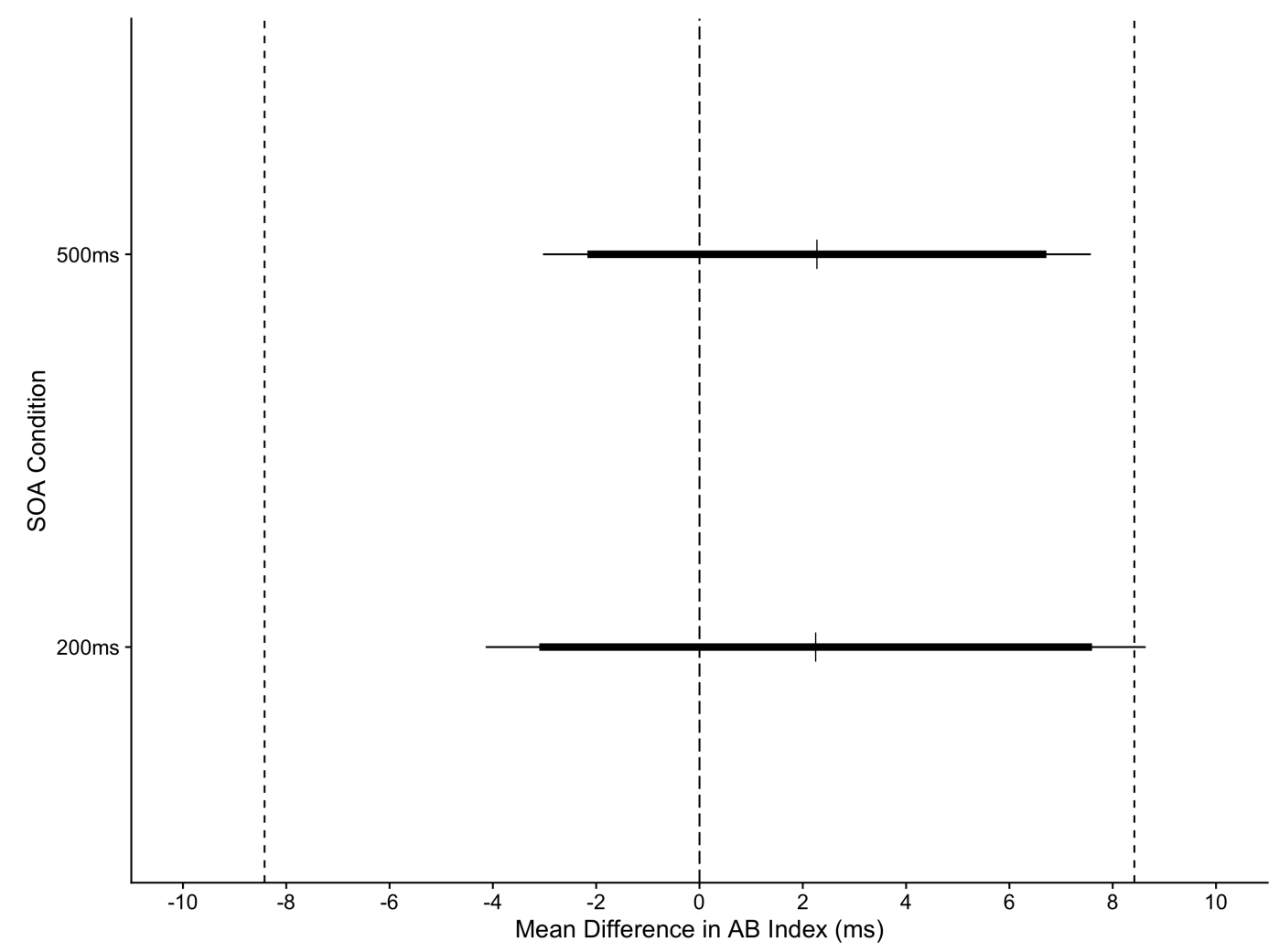

Figure 4. The thin vertical lines show the mean difference in attentional bias index between daily and non-daily smokers in each SOA condition. The thick horizontal black lines represent the $90 \%$ confidence interval for the two one-sided test procedure. The thin horizontal black lines represent the $95 \%$ confidence interval. The dashed vertical lines represent the equivalence boundaries in raw scores.

\section{Exploratory Analyses: Including trial type as an additional IV}

In our preregistration protocol, we focused on the attentional bias index as our outcome for confirmatory analyses, calculating it from the difference between smoking and neutral trials. While there were no meaningful differences between smoking groups, both peer-reviewers questioned whether participants first showed an attentional bias effect towards smoking images. Therefore, we performed exploratory analyses where we included trial type as an additional within-subjects IV instead of calculating the difference in RT 
between each condition.

We used a 2x2x2 mixed ANOVA using RT as our DV, trial type and SOA as within-subject IVs, and smoking group as a between-subjects IV. The only significant effect was $\operatorname{SOA}\left(F(1,164)=13.03, p<.001, \hat{\eta}_{G}^{2}=.002\right)$, which in isolation is not theoretically meaningful to us. None of the other effects were statistically significant.

Although there were no significant effects including trial type, we quantified whether participants showed an attentional bias effect towards smoking images using the persons as effect sizes approach (Grice et al., 2020). Instead of a blanket mean difference between groups or conditions, you can quantify how many participants behaved consistent with theoretical predictions. In this context, we can ask how many participants showed faster RTs to smoking trials compared to non-smoking trials.

For each participant, we coded whether the difference in RT was negative (faster responses to non-smoking images) or positive (faster responses to smoking images), then calculated the percentage showing a positive effect for each smoking group and SOA condition. Participants rarely deviated from 50\% showing the expected faster responses to smoking images. $50 \%$ of daily smokers in the $200 \mathrm{~ms}$ and $52.83 \%$ in the $500 \mathrm{~ms}$ SOA condition showed faster responses to smoking images. $53.33 \%$ of non-daily smokers in the $200 \mathrm{~ms}$ SOA condition showed faster responses to smoking images, while $43.33 \%$ responded faster to smoking images in the 500ms SOA condition, suggesting more participants responded faster to non-smoking images. We visualised these results in Figure 5 where each line represents a participant and the colour shows whether they responded faster to smoking or non-smoking images for each SOA condition and smoking group. Collectively, these exploratory analyses suggest participants did not display the predicted attentional bias effect towards smoking images. 


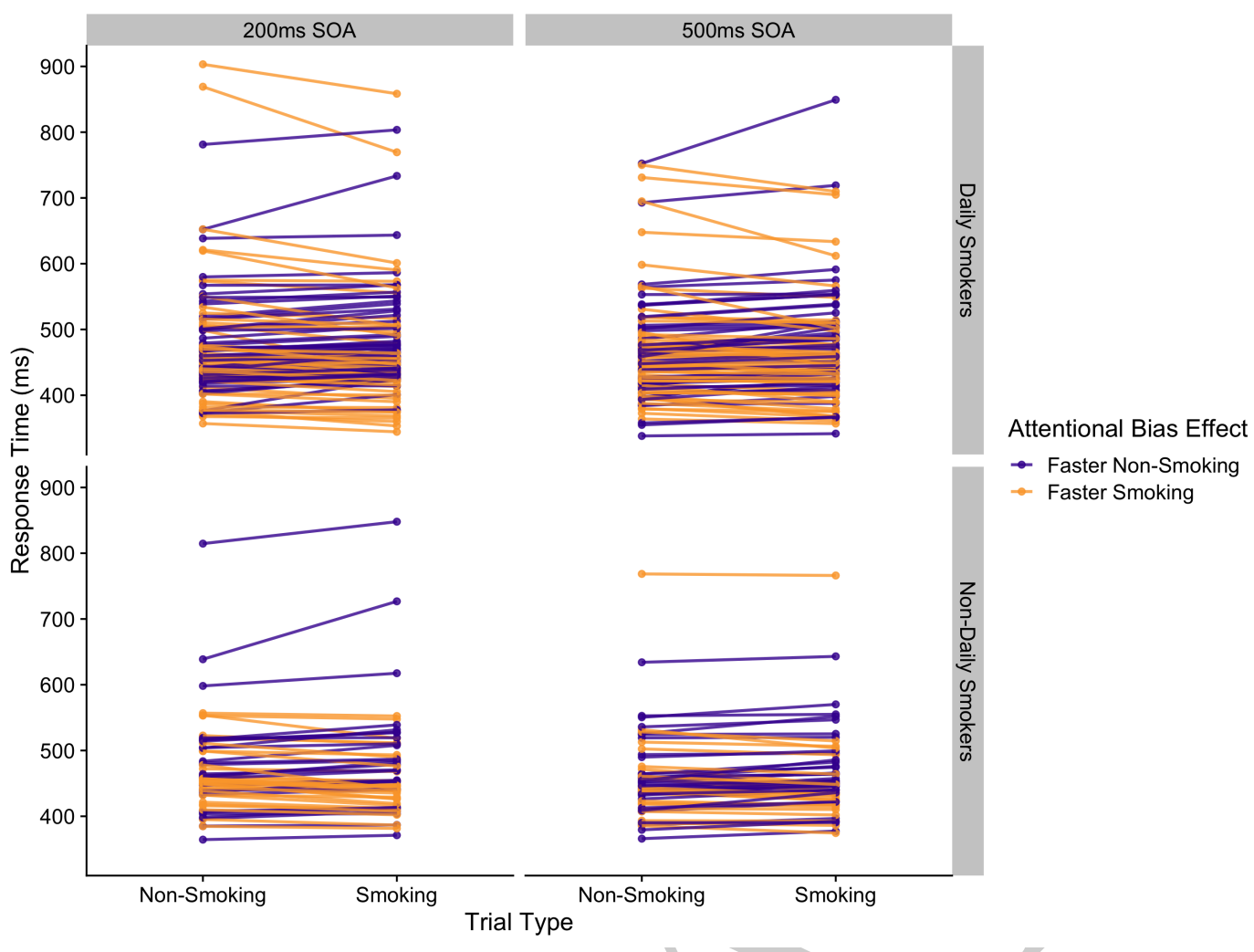

Figure 5. A dot plot visualising whether each participant showed the predicted attentional bias effect towards smoking images. Each line represents one participant where their average RT to non-smoking and smoking images is connected. Positive colour-coded slopes show participants who responded faster to non-smoking images while negative colour-coded slopes show participants who responded faster to smoking images. Each panel represents the combination of smoking group and SOA condition.

\section{Exploratory Analyses: Visual Probe Task Reliability}

We calculated Cronbach's alpha for the attentional bias index across the 16 stimulus pairs which was poor for both the $200 \mathrm{~ms}(\alpha=.29,95 \% \mathrm{CI}=[.00, .58])$ and $500 \mathrm{~ms}(\alpha=.19$, $95 \% \mathrm{CI}=[.00, .42])$ SOA conditions.

We reported internal consistency estimates for comparison with previous studies, but they assume the items or trials are presented in the same order (Parsons et al., 2019). As cognitive tasks randomise trials, internal consistency may not be the best approach. An 
alternative is a permutation approach to calculating split-half reliability (Parsons, 2020). This randomly splits the data set into two halves many times and calculates the average correlation between each half. Using 5000 iterations, poor reliability was also reflected in the split-half estimate (corrected using the Spearman-Brown formula) for the 200ms ( $r=.56$, $95 \% \mathrm{CI}=[.37, .7])$ and $500 \mathrm{~ms}(r=.47,95 \% \mathrm{CI}=[.27, .62])$ SOA conditions.

\section{Discussion}

We hypothesised that non-daily smokers would display greater attentional bias towards smoking cues than daily smokers. Some studies found that non-daily smokers exhibited greater attentional bias (Bradley et al., 2003; Hogarth et al., 2003; Mogg et al., 2005), whereas others found that daily smokers displayed greater attentional bias (Chanon et al., 2010; Vollstädt-Klein et al., 2011; Zack et al., 2001). Using traditional methods that calculate an attentional bias index from average differences in RT, there were no significant differences, and using equivalence testing showed there was no meaningful difference in attentional bias in daily and non-daily smokers.

We may have found null results as previous research could have problems with inflated effect sizes due to low statistical power. The previous largest sample was 51 smokers in Vollstädt-Klein et al. (2011). Splitting these into 25 and 26 participants, a sensitivity power analysis indicates that this sample size would be sensitive to detect effect sizes of Cohen's d $=0.80($ alpha $=.05$, beta $=.20)$. Incidentally, Schäfer and Schwarz $(2019)$ showed that the median Cohen's d in a random selection of 684 non-pre-registered articles was 0.80 . In the long-run, our study would have $99.80 \%$ power to detect an effect size of 0.80 . Therefore, it is unlikely the effect size between daily and non-daily smokers is this large, or we would have had enough power to detect it. Our study had the largest known sample size to investigate attentional bias with 60 non-daily smokers and 106 daily smokers. A sensitivity power analysis shows that this was sensitive to detect effect sizes of Cohen's $d=0.46$. Our study was sensitive to detect an effect size of almost half the size of Vollstädt-Klein et al. (2011). 
Our results were statistically equivalent to zero, meaning there may not be a meaningful difference in attentional bias between smoking groups, at least in its current implementation where the effect is assumed to represent stable trait-like group differences.

Contemporary theories suggest attentional bias may not be a trait-like phenomenon that can produce stable differences between groups. Field et al. (2016) suggested that attentional bias varies depending on how substance cues are being evaluated. The theory suggests that rather than being a stable trait between groups, it fluctuates with the incentive value of a cue which makes within-group differences more important. Begh et al. (2016) found that laboratory measures like the visual probe task did not predict smoking behaviour in the real-world. However, ecological momentary assessment of craving and awareness of smoking cues did predict smoking behaviour. Therefore, the null results in our study may be a product of the fluctuating nature of attentional bias (Field et al., 2016). In smaller samples, attentional bias could fluctuate one way or the other, but in larger samples like our study, the differences could cancel out and converge to a mean difference around zero. Therefore, future research may benefit from investigating which factors affect the momentary evaluation of substance cues and the subsequent expression of attentional bias.

Using the visual probe task to measure factors that affect the momentary evaluation of substance cues may be problematic though. There are vocal critics of the task due to its questionable level of internal consistency (Ataya et al., 2012; Schmukle, 2005; Waechter et al., 2014). Our study also had suboptimal levels of internal consistency and split-half reliability. Researchers rarely report the reliability of cognitive tasks unless it is the main focus of the article (Parsons et al., 2019), which means it is difficult to assess how reliable the tasks were in previous smoking research. Experimental measures are designed to produce reliable differences between groups or condition, not consistently rank individuals (Hedge et al., 2018). This means if researchers plan to use the visual probe task across multiple measurements - such as in cognitive bias modification or the evaluation of substance cues - 
then its poor reliability is problematic. Future research should use eye-tracking as a direct measure of attentional bias as it produces larger effect sizes (Field et al., 2009), has higher internal consistency (Price et al., 2015), and higher criterion validity (Soleymani et al., 2020).

\section{Limitations}

Our sample may have been more diverse than typical undergraduates in age and education, but it still contained predominantly white participants. Non-daily smoking is more prevalent in ethnic minority groups (Fagan \& Rigotti, 2009; Levy et al., 2009) and the health implications of smoking disproportionately affect non-white smokers (St.Helen et al., 2019). Therefore, future research would benefit from recruiting a larger proportion of non-white smokers for the results to generalise beyond mostly white smokers.

The online nature of the study meant participants' smoking levels could not be verified objectively using measures like Carbon Monoxide (Wray et al., 2016), but Ramo et al. (2011) demonstrated that smoking-related information collected online has good reliability and validity. Relatedly, as participants completed the study online, there was no control over their smoking behaviour before and during the study. This lead to idiosyncrasies as some smokers reported to smoke while they were completing the study. Although this may represent a more naturalistic environment for the smokers, our study had less control over smokers' deprivation levels.

\section{Conclusion}

The purpose of our study was investigate the conflict in attentional bias results between daily and non-daily smokers. We expected non-daily smokers to show greater attentional bias towards smoking images than daily smokers. Greater attentional bias in non-daily smokers would have helped to explain why they find it difficult to quit smoking while showing fewer signs of nicotine dependence. However, there were no significant effects, and using equivalence testing, we found that there was no meaningful difference in 
384

attentional bias between daily and non-daily smokers. The results can be interpreted in line with contemporary theories of attentional bias where there may not be stable trait-level differences between smoking groups in attentional bias. Future research should focus on investigating how attentional bias fluctuates over time using more reliable measures than the visual probe task.

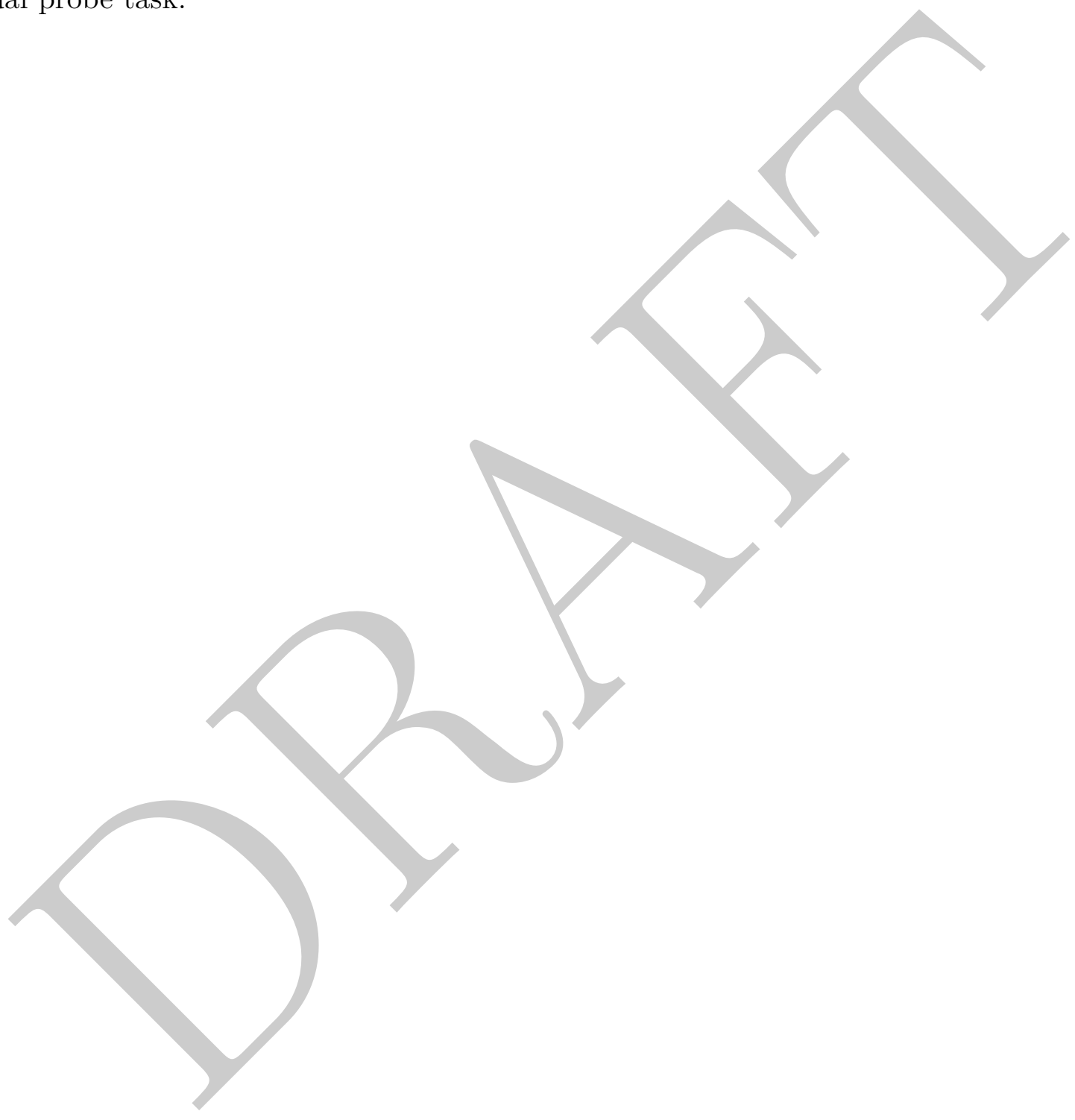




\section{CRediT Contributions}

\section{Disclosures}

Conceptualization (JEB, RJ, NW); Methodology (JEB, RJ, NW); Formal analysis (JEB); Investigation (JEB); Data curation (JEB); Writing - original draft (JEB); Writing Review \& editing (JEB); Supervision (RJ, NW).

\section{Data, code, and materials}

The data and code to reproduce these analyses are available on the OSF (https://osf.io/am9hd/). The visual probe task was created in Gorilla and the task can be found using the open materials page (http://gorilla.sc/openmaterials/85021)

\section{R Package Acknowledgements}

The results were created using $\mathrm{R}$ (Version 4.1.3; R Core Team, 2020) and the R-packages afex (Version 1.0.1; Singmann et al., 2020), cowplot (Version 1.1.1; Wilke, 2019), dplyr (Version 1.0.10; Wickham et al., 2020), ggplot2 (Version 3.3.5; Wickham, 2016), janitor (Version 2.1.0; Firke, 2019), papaja (Version 0.1.1; Aust \& Barth, 2020), psych (Version 2.2.3; Revelle, 2019), pwr (Version 1.3.0; Champely, 2020), readr (Version 2.1.2; Wickham et al., 2018), shiny (Version 1.7.1; Chang et al., 2020), splithalf (Version 0.8.2; Parsons, 2020), stringr (Version 1.4.0; Wickham, 2019), tibble (Version 3.1.6; Müller \& Wickham, 2020), tidyr (Version 1.2.0; Wickham \& Henry, 2020), tinylabels (Version 0.2.3; Barth, 2022), and TOSTER (Version 0.4.0; Lakens, 2017). 


\section{References}

Allen, M., Poggiali, D., Whitaker, K., Marshall, T. R., \& Kievit, R. A. (2019). Raincloud plots: A multi-platform tool for robust data visualization. Wellcome Open Research, 4, 63. https://doi.org/10.12688/wellcomeopenres.15191.1

Anwyl-Irvine, A., Massonnié, J., Flitton, A., Kirkham, N., \& Evershed, J. (2019). Gorilla in our Midst: An online behavioral experiment builder. Behavior Research Methods, 52, 388-407. https://doi.org/10.1101/438242

Ataya, A. F., Adams, S., Mullings, E., Cooper, R. M., Attwood, A. S., \& Munafò, M. R. (2012). Internal reliability of measures of substance-related cognitive bias. Drug and Alcohol Dependence, 121(1), 148-151. https://doi.org/10.1016/j.drugalcdep.2011.08.023

Aust, F., \& Barth, M. (2020). papaja: Create APA manuscripts with R Markdown. https://github.com/crsh/papaja

Barth, M. (2022). tinylabels: Lightweight variable labels. https://cran.r-project.org/package=tinylabels

Bartlett, J. E. (2020). Daily and Non-daily Smokers: A Profile of Drive and Cognitive Control Mechanisms [PhD thesis, Coventry University]. https://thesiscommons.org/h9gpe/

Baschnagel, J. S. (2013). Using mobile eye-tracking to assess attention to smoking cues in a naturalized environment. Addictive Behaviors, 38(12), 2837-2840. https://doi.org/10.1016/j.addbeh.2013.08.005

Begh, R., Smith, M., Ferguson, S. G., Shiffman, S., Munafò, M. R., \& Aveyard, P. (2016). Association between smoking-related attentional bias and craving measured in the clinic and in the natural environment. Psychology of Addictive Behaviors, 30(8), 868-875. https://doi.org/10.1037/adb0000231

Bogdanovica, I., Godfrey, F., McNeill, A., \& Britton, J. (2011). Smoking prevalence in the European Union: A comparison of national and transnational prevalence 
survey methods and results. Tobacco Control, 20(1), 1-9. https://doi.org/10.1136/tc.2010.036103

Bradley, B. P., Mogg, K., Wright, T., \& Field, M. (2003). Attentional bias in drug dependence: Vigilance for cigarette-related cues in smokers. Psychology of Addictive Behaviors, 17(1), 66-72. https://doi.org/10.1037/0893-164X.17.1.66

Champely, S. (2020). Pwr: Basic functions for power analysis. https://CRAN.R-project.org/package $=$ pwr

Chang, W., Cheng, J., Allaire, J., Xie, Y., \& McPherson, J. (2020). Shiny: Web application framework for $r$. https://CRAN.R-project.org/package=shiny

Chanon, V. W., Sours, C. R., \& Boettiger, C. A. (2010). Attentional bias toward cigarette cues in active smokers. Psychopharmacology, 212(3), 309-320. https://doi.org/10.1007/s00213-010-1953-1

Clifford, S., \& Jerit, J. (2014). Is There a Cost to Convenience? An Experimental Comparison of Data Quality in Laboratory and Online Studies. Journal of Experimental Political Science, 1(2), 120-131. https://doi.org/10.1017/xps.2014.5

Ehrman, R. N., Robbins, S. J., Bromwell, M. A., Lankford, M. E., Monterosso, J. R., \& O'Brien, C. P. (2002). Comparing attentional bias to smoking cues in current smokers, former smokers, and non-smokers using a dot-probe task. Drug and Alcohol Dependence, 67(2), 185-191. https://doi.org/10.1016/S0376-8716(02)00065-0

Fagan, P., \& Rigotti, N. A. (2009). Light and intermittent smoking: The road less traveled. Nicotine \& Tobacco Research, 11 (2), 107-110. https://doi.org/10.1093/ntr/ntn015

Fagerström, K. (2012). Determinants of Tobacco Use and Renaming the FTND to the Fagerström Test for Cigarette Dependence. Nicotine \& Tobacco Research, 14 (1), 75-78. https://doi.org/10.1093/ntr/ntr137

Field, M., \& Cox, W. M. (2008). Attentional bias in addictive behaviors: A review of 
its development, causes, and consequences. Drug and Alcohol Dependence, 97(1-2), 1-20. https://doi.org/10.1016/j.drugalcdep.2008.03.030

Field, M., Munafò, M. R., \& Franken, I. H. A. (2009). A meta-analytic investigation of the relationship between attentional bias and subjective craving in substance abuse. Psychological Bulletin, 135(4), 589-607. https://doi.org/10.1037/a0015843

Field, M., Werthmann, J., Franken, I., Hofmann, W., Hogarth, L., \& Roefs, A. (2016). The role of attentional bias in obesity and addiction. Health Psychology, 35(8), 767-780. https://doi.org/10.1037/hea0000405

Firke, S. (2019). Janitor: Simple tools for examining and cleaning dirty data. https://CRAN.R-project.org/package=janitor

Grice, J. W., Medellin, E., Jones, I., Horvath, S., McDaniel, H., O’lansen, C., \& Baker, M. (2020). Persons as Effect Sizes. Advances in Methods and Practices in Psychological Science, 3(4), 443-455. https://doi.org/10.1177/2515245920922982 Heatherton, T. F., Kozlowski, L. T., Frecker, R. C., \& Fagerström, K.-O. (1991). The Fagerström Test for Nicotine Dependence: A revision of the Fagerstrom Tolerance Questionnaire. British Journal of Addiction, 86(9), 1119-1127. https://doi.org/10.1111/j.1360-0443.1991.tb01879.x

Hedge, C., Powell, G., \& Sumner, P. (2018). The reliability paradox: Why robust cognitive tasks do not produce reliable individual differences. Behavior Research Methods, 50(3), 1166-1186. https://doi.org/10.3758/s13428-017-0935-1

Hogarth, L. C., Mogg, K., Bradley, B. P., Duka, T., \& Dickinson, A. (2003). Attentional orienting towards smoking-related stimuli: Behavioural Pharmacology, 14 (2), 153-160. https://doi.org/10.1097/00008877-200303000-00007

Kang, O.-S., Chang, D.-S., Jahng, G.-H., Kim, S.-Y., Kim, H., Kim, J.-W., Chung, S.-Y., Yang, S.-I., Park, H.-J., Lee, H., \& Chae, Y. (2012). Individual differences in smoking-related cue reactivity in smokers: An eye-tracking and fMRI study. Progress in Neuro-Psychopharmacology and Biological Psychiatry, 38(2), 285-293. 
https://doi.org/10.1016/j.pnpbp.2012.04.013

Kotz, D., Fidler, J., \& West, R. (2012). Very low rate and light smokers: Smoking patterns and cessation-related behaviour in England, 2006-11: Very low rate and light smokers. Addiction, 107(5), 995-1002. https://doi.org/10.1111/j.1360-0443.2011.03739.x

Lakens, D. (2017). Equivalence tests: A practical primer for t-tests, correlations, and meta-analyses. Social Psychological and Personality Science, 1, 1-8. https://doi.org/10.1177/1948550617697177

Lakens, D., Scheel, A. M., \& Isager, P. M. (2018). Equivalence Testing for Psychological Research: A Tutorial. Advances in Methods and Practices in Psychological Science, 1(2), 259-269. https://doi.org/10.1177/2515245918770963 Lang, P. J., Bradley, M. M., \& Cuthbert, B. N. (2008). International affective picture system (IAPS): Affective ratings of pictures and instruction manual. Technical Report A-8. Gainesville: University of Florida.

Levy, D. E., Biener, L., \& Rigotti, N. A. (2009). The natural history of light smokers: A population-based cohort study. Nicotine \& Tobacco Research, 11(2), 156-163. https://doi.org/10.1093/ntr/ntp011

Mogg, K., Bradley, B. P., Field, M., \& De Houwer, J. (2003). Eye movements to smoking-related pictures in smokers: Relationship between attentional biases and implicit and explicit measures of stimulus valence. Addiction, 98(6), 825-836. https://doi.org/10.1046/j.1360-0443.2003.00392.x

Mogg, K., Field, M., \& Bradley, B. P. (2005). Attentional and approach biases for smoking cues in smokers: An investigation of competing theoretical views of addiction. Psychopharmacology, 180(2), 333-341. https://doi.org/10.1007/s00213-005-2158-x Müller, K., \& Wickham, H. (2020). Tibble: Simple data frames. https://CRAN.R-project.org/package=tibble 
Parsons, S. (2020). Splithalf; robust estimates of split half reliability. https://doi.org/10.6084/m9.figshare.5559175.v5

Parsons, S., Kruijt, A.-W., \& Fox, E. (2019). Psychological Science Needs a Standard Practice of Reporting the Reliability of Cognitive-Behavioral Measurements. Advances in Methods and Practices in Psychological Science, 2(4), 378-395. https://doi.org/10.1177/2515245919879695

Pennington, C. R., Jones, A., Bartlett, J. E., Copeland, A., \& Shaw, D. J. (2021). Raising the bar: Improving methodological rigour in cognitive alcohol research. Addiction, 116(11), 3243-3251. https://doi.org/https://doi.org/10.1111/add.15563

Price, R. B., Kuckertz, J. M., Siegle, G. J., Ladouceur, C. D., Silk, J. S., Ryan, N. D., Dahl, R. E., \& Amir, N. (2015). Empirical Recommendations for Improving the Stability of the Dot-Probe Task in Clinical Research. Psychological Assessment, 27(2), 365-376. https://doi.org/10.1037/pas0000036

R Core Team. (2020). R: A language and environment for statistical computing. $\mathrm{R}$ Foundation for Statistical Computing. https://www.R-project.org/

Ramo, D. E., Hall, S. M., \& Prochaska, J. J. (2011). Reliability and validity of self-reported smoking in an anonymous online survey with young adults. Health Psychology, 30(6), 693-701. https://doi.org/10.1037/a0023443

Revelle, W. (2019). Psych: Procedures for psychological, psychometric, and personality research. Northwestern University. https://CRAN.R-project.org/package=psych

Salemink, E., Hout, M. A. van den, \& Kindt, M. (2007). Selective attention and threat: Quick orienting versus slow disengagement and two versions of the dot probe task. Behaviour Research and Therapy, 45(3), 607-615. https://doi.org/10.1016/j.brat.2006.04.004

Schäfer, T., \& Schwarz, M. A. (2019). The Meaningfulness of Effect Sizes in 
Psychological Research: Differences Between Sub-Disciplines and the Impact of Potential Biases. Frontiers in Psychology, 10, 1-13. https://doi.org/10.3389/fpsyg.2019.00813

Schmukle, S. C. (2005). Unreliability of the dot probe task. European Journal of Personality, 19(7), 595-605. https://doi.org/10.1002/per.554

Shiffman, S. (2009). Light and intermittent smokers: Background and perspective. Nicotine $\&$ Tobacco Research, 11 (2), 122-125. https://doi.org/10.1093/ntr/ntn020 Shiffman, S., Dunbar, M. S., Li, X., Scholl, S. M., Tindle, H. A., Anderson, S. J., \& Ferguson, S. G. (2014). Smoking Patterns and Stimulus Control in Intermittent and Daily Smokers. PLoS ONE, 9(3), 1-14. https://doi.org/10.1371/journal.pone.0089911

Shiffman, S., Dunbar, M. S., Scholl, S. M., \& Tindle, H. A. (2012). Smoking motives of daily and non-daily smokers: A profile analysis. Drug and Alcohol Dependence, 126 (3), 362-368. https://doi.org/10.1016/j.drugalcdep.2012.05.037

Shiffman, S., Tindle, H., Li, X., Scholl, S., Dunbar, M., \& Mitchell-Miland, C. (2012). Characteristics and smoking patterns of intermittent smokers. Experimental and Clinical Psychopharmacology, 20(4), 264-277. https://doi.org/10.1037/a0027546

Simonsohn, U. (2015). Small Telescopes: Detectability and the Evaluation of Replication Results. Psychological Science, 26 (5), 559-569. https://doi.org/10.1177/0956797614567341

Singmann, H., Bolker, B., Westfall, J., Aust, F., \& Ben-Shachar, M. S. (2020). Afex: Analysis of factorial experiments. https://CRAN.R-project.org/package=afex

Soleymani, A., Ivanov, Y., Mathot, S., \& Jong, P. J. de. (2020). Free-viewing multi-stimulus eye tracking task to index attention bias for alcohol versus soda cues: Satisfactory reliability and criterion validity. Addictive Behaviors, 100, 106117. https://doi.org/10.1016/j.addbeh.2019.106117

St.Helen, G., Benowitz, N. L., Ahluwalia, J. S., Tyndale, R. F., Addo, N., Gregorich, 
S. E., Pérez-Stable, E. J., \& Cox, L. S. (2019). Black Light Smokers: How Nicotine Intake and Carcinogen Exposure Differ Across Various Biobehavioral Factors. Journal of the National Medical Association, 111(5), 509-520. https://doi.org/10.1016/j.jnma.2019.04.004

Tindle, H. A., \& Shiffman, S. (2011). Smoking Cessation Behavior Among Intermittent Smokers Versus Daily Smokers. American Journal of Public Health, 101 (7), e1-e3. https://doi.org/10.2105/AJPH.2011.300186

Tong, E. K., Ong, M. K., Vittinghoff, E., \& Pérez-Stable, E. J. (2006). Nondaily Smokers Should Be Asked and Advised to Quit. American Journal of Preventive Medicine, 30(1), 23-30. https://doi.org/10.1016/j.amepre.2005.08.048

Vollstädt-Klein, S., Loeber, S., Winter, S., Leménager, T., Goltz, C. von der, Dinter, C., Koopmann, A., Wied, C., Winterer, G., \& Kiefer, F. (2011). Attention Shift towards Smoking Cues Relates to Severity of Dependence, Smoking Behavior and Breath Carbon Monoxide. European Addiction Research, 17(4), 217-224. https://doi.org/10.1159/000327775

Waechter, S., Nelson, A. L., Wright, C., Hyatt, A., \& Oakman, J. (2014). Measuring Attentional Bias to Threat: Reliability of Dot Probe and Eye Movement Indices. Cognitive Therapy and Research, 38(3), 313-333. https://doi.org/10.1007/s10608-013-9588-2

Wickham, H. (2016). ggplot2: Elegant graphics for data analysis. Springer-Verlag New York. https://ggplot2.tidyverse.org

Wickham, H. (2019). Stringr: Simple, consistent wrappers for common string operations. https://CRAN.R-project.org/package=stringr

Wickham, H., François, R., Henry, L., \& Müller, K. (2020). Dplyr: A grammar of data manipulation. https://CRAN.R-project.org/package=dplyr

Wickham, H., \& Henry, L. (2020). Tidyr: Tidy messy data. https://CRAN.R-project.org/package=tidyr 
Wickham, H., Hester, J., \& Francois, R. (2018). Readr: Read rectangular text data. https://CRAN.R-project.org/package $=$ readr

Wilke, C. O. (2019). Cowplot: Streamlined plot theme and plot annotations for 'ggplot2'. https://CRAN.R-project.org/package=cowplot

Wray, J. M., Gass, J. C., Miller, E. I., Wilkins, D. G., Rollins, D. E., \& Tiffany, S. T. (2016). A Comparative Evaluation of Self-Report and Biological Measures of Cigarette Use in Non-Daily Smokers. Psychological Assessment, 28(9), 1043-1050. https://doi.org/10.1037/pas0000227

Zack, M., Belsito, L., Scher, R., Eissenberg, T., \& Corrigall, W. A. (2001). Effects of abstinence and smoking on information processing in adolescent smokers. Psychopharmacology, 153(2), 249-257. https://doi.org/10.1007/s002130000552 